Martens, Matthias; Asbrand, Barbara; Spieß, Christian

\title{
Lernen mit Dingen - Prozesse zirkulierender Referenz im Unterricht
}

Zeitschrift für interpretative Schul- und Unterrichtsforschung 4 (2015) 1, S. 48-65

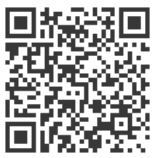

Quellenangabe/ Reference:

Martens, Matthias; Asbrand, Barbara; Spieß, Christian: Lernen mit Dingen - Prozesse zirkulierender Referenz im Unterricht - In: Zeitschrift für interpretative Schul- und Unterrichtsforschung 4 (2015) 1, S. 48-65 - URN: urn:nbn:de:0111-pedocs-153456 - DOI: 10.25656/01:15345

https://nbn-resolving.org/urn:nbn:de:0111-pedocs-153456

https://doi.org/10.25656/01:15345

in Kooperation mit / in cooperation with:

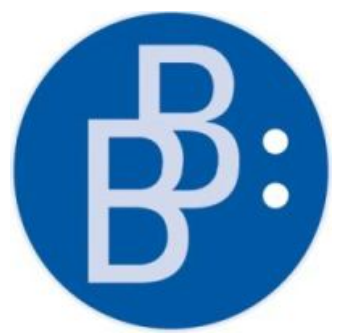

https://www.budrich.de

\section{Nutzungsbedingungen}

Gewährt wird ein nicht exklusives, nicht übertragbares, persönliches und beschränktes Recht auf Nutzung dieses Dokuments. Dieses Dokument ist ausschließlich für den persönlichen, nicht-kommerziellen Gebrauch bestimmt. Die Nutzung stellt keine Übertragung des Eigentumsrechts an diesem Dokument dar und gilt vorbehaltlich der folgenden Einschränkungen: Auf sämtlichen Kopien dieses Dokuments müssen alle Urheberrechtshinweise und sonstigen Hinweise auf gesetzlichen Schutz beibehalten werden. Sie dürfen dieses Dokument nicht in irgendeiner Weise abändern, noch dürfen Sie dieses Dokument für öffentliche oder kommerzielle Zwecke vervielfältigen, öffentlich ausstellen, aufführen, vertreiben oder anderweitig nutzen.

Mit der Verwendung dieses Dokuments erkennen Sie die Nutzungsbedingungen an.

\section{Terms of use}

We grant a non-exclusive, non-transferable, individual and limited right to using this document.

This document is solely intended for your personal, non-commercial use. Use of this document does not include any transfer of property rights and it is conditional to the following limitations: All of the copies of this documents must retain all copyright information and other information regarding legal protection. You are not allowed to alter this document in any way, to copy it for public or commercial purposes, to exhibit the document in public, to perform, distribute or otherwise use the document in public.

By using this particular document, you accept the above-stated conditions of use.

\section{Kontakt / Contact:}

\section{peDOCS}

DIPF | Leibniz-Institut für Bildungsforschung und Bildungsinformation Informationszentrum (IZ) Bildung

E-Mail: pedocs@dipf.de

Internet: www.pedocs.de

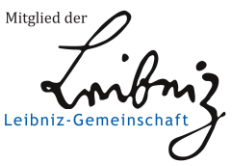




\section{Inhalt}

4. Jahrgang 2015

\section{ZISU}

\section{Zeitschrift für interpretative Schul- und Unterrichtsforschung}

\section{Editorial}

Ulrich Gebhard, Räume, Dinge und schulisches Wissen.

Merle Hummrich, Eine Einführung

Kerstin Rabenstein,

Sabine Reh

\section{Thementeil}

Georg Breidenstein

Vincent und die „Apotheke“ - oder: die Didaktik des

Materials

Thomas Grunau, Mirja Kekeritz

„Verdammtes Ding, dich könn' wir dann erforschen!“ Perspektiven auf kindliche Auseinandersetzungen mit den Dingen in institutionellen Kontexten

Matthias Martens, Lernen mit Dingen - Prozesse zirkulierender Referenz im Unterricht

Christian Spieß

Julia Demmer

Artefakte und Wissensformen in biografischen

Selbstpräsentationen von Zeitzeug_innen.

Fallrekonstruktion Schule

Jutta Wiesemann,

„Education in a Box“ Die Herstellung schulischer

\section{Allgemeiner Teil}

Wiebke Bobeth-

Neumann

Anne Niessen
Angebote zur Qualifizierung für den

Schulleitungsberuf: Deutungsmuster von Lehrkräften auf dem Weg ins Schulleitungsamt

Musikpädagogische Perspektiven auf Heterogenität und die Ambivalenz der Anerkennung. Ergebnisse einer qualitativen Interviewstudie mit Lehrenden des Programms ,Jedem Kind ein Instrument“ 
Sammy Wafi, Markus Wirtz

\section{Rezensionen}

Svenja Strauß

Till-Sebastian Idel
Visualisierungskompetenz in Deutsch und

119 Mathematik aus Sicht von Expertinnen und Experten der Lehr-/Lernforschung und Fachdidaktik

Kade, Jochen/Nolda, Sigrid/Dinkelaker, Jörg/

Herrle, Matthias (Hrsg.) (2014): Videographische

Kursforschung. Empirie des Lehrens und Lernens

Erwachsener. Stuttgart: Kohlhammer Verlag

Zeitler, Sigrid/Heller, Nina/Asbrand, Barbara (2012):

145

Bildungsstandards in der Schule. Eine rekonstruktive Studie zur Implementation der Bildungsstandards. Münster/New York/München/Berlin: Waxmann. ISBN 978-3-8309-2664-1. 


\title{
Lernen mit Dingen - Prozesse zirkulierender Referenz im Unterricht
}

\begin{abstract}
Zusammenfassung
Dinge sind auf vielfältige Weise in unterrichtliche Lernprozesse involviert und für diese bedeutsam. In der empirischen Analyse von Unterrichtsvideografien mit der Dokumentarischen Methode zeigte sich praxeologisch, dass die Theoriefigur der „zirkulierenden Referenz“ (Latour) geeignet ist, zur Ausdifferenzierung einer gegenstandstheoretischen Beschreibung unterrichtlicher Lernprozesse und ihrer Materialität beizutragen. An Beispielen aus dem Geschichtsunterricht zeigen wir, welche besondere Bedeutung die Materialität von Quellen für das historische Lernen entfaltet. Die rekonstruierten Erkenntnisprozesse der Schülerinnen und Schüler in Assoziation mit Sachquellen, d.h. mit authentischen Gegenständen aus der Vergangenheit, können mit Latour als Tätigkeiten des Abstrahierens beschrieben werden.

Schlagwörter: Materialität im Unterricht, Lernen, Erkenntnis, Dokumentarische Methode, Quellenarbeit, historisches Lernen, Geschichtsunterricht, Akteur-Netzwerk-Theorie
\end{abstract}

\section{Learning with things - processes of circulating references in teaching}

In many different ways, material objects are relevant to learning in the classroom. As we were analyzing videotaped lessons with the Documentary Method, we found that Bruno Latour's notion of "circulating reference" can describe the way in which objects are a part of school learning. Using examples from history lessons, we demonstrate the impact that the use of authentic artifacts can have on historical understanding. As students construct meaning based on the interaction with authentic and palpable historical evidence they are performing what Latour has called an act of abstraction.

Keywords: materiality and teaching, documentary method, working with sources, historical learning, actor-network theory

\section{Einleitung: Dinge im Unterricht}

Dinge nehmen in der erziehungswissenschaftlichen Reflexion auf Schule und Unterricht bisher einen eher untergeordneten Stellenwert ein, obwohl ihre Bedeutung für Sozialisation, Erziehung und Bildung bereits vielfach herausgestellt wurde (Langeveld 1955; Meyer-Drawe 1999; Stieve 2008; Nohl 2011). Der Bedeutung der Dinge für den Unterricht tragen in den letzten Jahren zunehmend empirische Untersuchungen Rechnung. Verschiedene ethnografische Studien zur Rolle der Dinge im Unterricht nehmen praxistheoretische Überlegungen zum Ausgang. Reckwitz (2003) betont, dass soziale Praktiken allgemein immer auch eine materielle Komponente enthalten: soziale Praktiken konstituieren sich demnach erst durch routinierte Bewegungen und Aktivitäten der menschlichen Körper sowie durch den „sinnhaften Gebrauch“ (ebd. S. 291) von Dingen durch die Menschen. Studien der ethnografischen Unterrichtsforschung konzentrieren sich entsprechend darauf, zu beschreiben, wie mit den Dingen interagiert wird und wel- 
che Funktion sie innerhalb der untersuchten Unterrichtssettings haben. So rekonstruieren Breidenstein (2006) sowie Kalthoff und Roehl (2011) die Wandtafel als Träger des zu lernenden Wissens. Durch schriftliches Festhalten wird es als „richtiges Wissen“ markiert und zugänglich gemacht. Rabenstein und Wienike (2013) zeigen, wie mit Hilfe eines Rechenrahmens nicht nur Rechenoperationen eingeübt, sondern auch der Arbeitsprozess einer Schülerin für die Lehrkraft sicht- und überprüfbar gemacht wird. Während sich Roehl (2012) auf die Frage konzentriert, inwiefern die „Dinge des Wissens“ der medialen Vermittlung und Repräsentation schulischen Wissens dienen, fokussieren Rabenstein und Wienike (2013) die „Dinge des Lernens“. Darunter verstehen sie die Gegenstände wie z.B. Schreibgeräte, Hefte, Bücher, Lernhilfen etc., mit denen die Lernenden im Unterricht hantieren. Die Untersuchungen zeigen, dass und wie die Dinge in die Praktiken involviert werden. Allerdings sind die Lernprozesse der Schülerinnen und Schüler, verstanden als ko-konstruktive Prozesse der Aneignung und Herstellung von Wissen und Können, nicht im Fokus der ethnografischen Studien. Einen anderen theoretischen Ansatz zur Beschreibung der Bedeutung von Dingen im Mathematikunterricht wählt Fetzer (2010). Als Weiterentwicklung der interpretativen Unterrichtsforschung (Krummheuer \& Naujok 1999) rekurriert sie auf die Akteur-Netzwerk-Theorie Latours (2005) und rekonstruiert das Lernen der Schülerinnen und Schüler in Anlehnung an Blumer (1969) als soziale Prozesse der Bedeutungsgenerierung, in die die Dinge involviert sind.

Bei der Analyse von Unterrichtsvideografien mit der Dokumentarischen Methode (Bohnsack 2007; 2009) gehen wir der Frage nach, wie unterrichtliche Lehr- und Lernprozesse und deren Bedingungen in ihrer Komplexität der empirischen Erforschung zugänglich gemacht werden können. Über die Untersuchung der verbalen Unterrichtsinteraktion hinaus ist hierfür auch eine Berücksichtigung der nonverbalen Interaktionen und der Interaktionen mit den Dingen in ihrem eigenlogischen AufeinanderbezogenSein notwendig. Praxeologisch, auf der Grundlage der empirischen Rekonstruktion der Materialität von Unterrichtsprozessen, erwies sich Latours Akteur-Netzwerk-Theorie (Latour 2005; 2002) in zweierlei Weise als ertragreich: Zum einen konnten wir die von Latour beschriebenen Formen der Assoziation von Menschen und Dingen in unsere Analyse von Unterrichtsvideografien integrieren und damit der Eigenlogik der Materialität des Unterrichts methodologisch und methodisch gerecht werden (Martens, Petersen \& Asbrand 2014). Zum anderen trägt Latours Konzept der „zirkulierenden Referenz“ (Latour 2002: 36ff) zur Ausdifferenzierung einer gegenstandstheoretischen Beschreibung von empirisch rekonstruierten unterrichtlichen Lernprozessen bei (Asbrand, Martens \& Petersen 2013).

Unter ,zirkulierender Referenz“ versteht Latour Prozesse der Generierung von wissenschaftlichem Wissen durch Forscherinnen und Forscher. Auf der Grundlage seiner ethnografischen Technik- und Wissenschaftsforschung kommt er zu dem Schluss, die Herstellung dieses Wissens basiere einerseits auf der Beobachtung und Analyse von (materiellen) Phänomenen in der Natur, häufig unter Zuhilfenahme technischer Geräte - also Dingen, die ebenfalls einen wesentlichen Anteil am Erkenntnisprozess haben. Andererseits entstehe neues Wissen durch die Referenz auf bereits vorhandenes und veröffentlichtes wissenschaftliches Wissen. Diesen Prozess der zirkulierenden Bezugnahme auf unterschiedliche materielle Dinge (die untersuchten Phänomene, technische 
Geräte, Bücher, Karten etc.) rekonstruiert Latour als eine Tätigkeit des Abstrahierens, in der, ausgehend von den beobachtbaren konkreten, zumeist materiellen Phänomenen der realen Welt, neues abstraktes, begriffliches Wissen entsteht. Er spricht von einer Tätigkeit, da er auf der Basis seiner Beobachtungen der wissenschaftlichen Erkenntnisprozesse die Genese von wissenschaftlichem Wissen notwendig mit dem praktischen Tun und dem Interagieren der Wissenschaftlerinnen und Wissenschaftler mit den Gegenständen verbunden sieht (ebd.).

In der empirischen Analyse von Unterrichtsvideografien zeigt sich praxeologisch, dass sich auch Prozesse der Wissensgenese im Unterricht als Prozess der zirkulierenden Referenz beobachten lassen, im Unterschied zur Herstellung wissenschaftlichen Wissens allerdings nicht nur als Tätigkeit des Abstrahierens, sondern auch als Tätigkeit des Konkretisierens (Asbrand, Martens \& Petersen 2013). So bezeichnen wir die rekonstruierten Unterrichtsprozesse, bei denen zwar zirkulierend auf Artefakte und vorhandenes Wissen Bezug genommen wird. Allerdings wird dabei nicht auf der Basis der Beschäftigung mit den Dingen neues Wissen generiert, sondern von den Schülerinnen und Schülern bereits gewusstes begriffliches Wissen mit Bezugnahme auf das Artefakt veranschaulicht. Während wir unsere theoretischen Überlegungen zum Lernen als Tätigkeit des Konkretisierens bereits an anderer Stelle veröffentlicht haben (ebd.), möchten wir mit diesem Beitrag zeigen, inwiefern sich empirisch rekonstruierte Lernprozesse, in die Dinge involviert sind, mit der Theoriefigur der ,zirkulierenden Referenz“ als Tätigkeiten des Abstrahierens beschreiben lassen.

Nach einer kurzen Darstellung des methodologischen und methodischen Rahmens zeigen wir Ergebnisse einer Rekonstruktion von Tätigkeiten des Abstrahierens an einem Beispiel aus dem Geschichtsunterricht, in dem gegenständliche Quellen zum Einsatz kommen. Abschließend reflektieren wir den unterrichtstheoretischen Ertrag der Rekonstruktion und der Bezugnahme auf die Akteur-Netzwerk-Theorie.

\section{Dokumentarische Unterrichtsforschung: Methodologie und Methode}

In unseren Forschungsprojekten der dokumentarischen Unterrichtsforschung (z.B. Petersen \& Asbrand 2014; Petersen 2015; Spieß 2014; Wettstädt \& Asbrand 2013; 2014), in denen es sowohl um die Rekonstruktion der Herstellung sozialer Ordnung des Unterrichts als auch um die Rekonstruktion von fachlichen Lehr- und Lernprozessen und deren Bedingungen geht, analysieren wir Unterrichtsvideografien mit Hilfe der Dokumentarischen Methode (Martens, Petersen \& Asbrand 2014). Im Anschluss an die Wissenssoziologie Mannheims (1980) ist die Dokumentarische Methode ein Verfahren zur Rekonstruktion von Prozessen der Wissensgenese. Zentral ist die Unterscheidung zweier Wissensformen. Während das kommunikative Wissen ein theoretisches, explizierbares Wissen bezeichnet, handelt es sich beim konjunktiven Wissen um ein implizites, atheoretisches Wissen, das der sozialen Praxis eingeschrieben ist und das Handeln von Menschen orientiert (ebd.: 211ff.; Bohnsack 2007: 59ff.). Bei der Erforschung von fachlichen Lern- bzw. Kompetenzerwerbsprozessen lassen sich aufgrund der Unterscheidung der beiden Wissensformen das erworbene Fachwissen der Schülerinnen und 
Schüler als theoretisches bzw. kommunikatives Wissen und die nicht-kognitiven Facetten von Kompetenz wie Routinen, Werthaltungen und Handlungsbereitschaften als konjunktives Wissen rekonstruieren (Martens \& Asbrand 2009). Gleichzeitig lässt sich durch diese Differenzierung auch der pädagogische Anspruch bzw. die pädagogische Programmatik des Unterrichts im Sinne eines kommunikativen Wissens von der Unterrichtspraxis und deren habituellen Strukturen im Sinne eines konjunktiven Wissens unterscheiden. Lehr- und Lernprozesse lassen sich auf der Basis von Unterrichtsvideografien dabei nicht nur aufgrund der diskursiven, sprachlichen Fähigkeiten der Akteure rekonstruieren, sondern auch als praktisches fachliches Können in der nonverbalen Interaktion und im Umgang mit den Dingen.

Ziel der dokumentarischen Interpretation ist im Rahmen mehrdimensionaler Fallvergleiche die Rekonstruktion derjenigen milieu-, gruppen- oder gegenstandsspezifischen konjunktiven Erfahrungsräume (Mannheim 1980: 230f.), in denen konjunktives Wissen generiert bzw. Kompetenzen angeeignet werden. Für die Unterrichtsforschung sind die professionsspezifischen oder berufsbiografischen Erfahrungen der Lehrkräfte und die milieu- oder geschlechtsspezifischen konjunktiven Erfahrungen der Kinder und Jugendlichen von Bedeutung. Außerdem fassen wir den Unterricht einer Schulklasse als einen gruppenspezifischen konjunktiven Erfahrungsraum und einzelne Unterrichtssequenzen als situationsspezifische bzw. gegenstandsbezogene konjunktive Erfahrungsräume auf, in denen sich Prozesse der Wissensgenese ereignen (Asbrand \& Nohl 2013).

Während die Frage, wie die Simultaneität und die Körperlichkeit sozialer Interaktion in die dokumentarische Interpretation einbezogen werden können, bereits in vorliegenden Arbeiten zur Videoanalyse bearbeitet wurde (z.B. Nentwig-Gesemann 2006; Nentwig-Gesemann \& Wagner-Willi 2007; Wagner-Willi 2008; Bohnsack 2009), ist die Analyse der Materialität von Interaktionen in methodologischer und methodischer Hinsicht bisher noch nicht geklärt. In unserer Forschungspraxis hat es sich als praktikabel erwiesen, die von Latour (2002: 211ff.) beschriebenen Formen der Assoziation von Menschen und Dingen in die sequenzielle Analyse der Dokumentarischen Methode $\mathrm{zu}$ integrieren, um die Bezugnahme und Interaktion der am Unterricht beteiligten Menschen mit Dingen aller Art in der empirischen Rekonstruktion des Unterrichts zu berücksichtigen (Asbrand, Martens \& Petersen 2013).

Hierfür ist von Bedeutung, dass die Theorie Latours anschlussfähig ist an die für die Dokumentarische Methode grundlegende Wissenssoziologie Mannheims. Beide Theoretiker sind an der Überwindung des Subjekt-Objekt-Gegensatzes der modernen Erkenntnistheorie interessiert und gehen von der Verbindung von Mensch und Dingen aus (Mannheim 1980: 164ff.; Latour 2002: 7ff.; dazu auch Schäffer 2001). Mit dem Begriff der Kontagion beschreibt Mannheim das unmittelbare Berührtsein zwischen Menschen sowie zwischen Menschen und der Natur bzw. den Dingen (Mannheim 1980: 125ff.). Für die Dokumentarische Methode wurde mit Fokus auf die Rekonstruktion von Prozessen der Wissensgenese bisher jedoch vor allem der Begriff der Konjunktion rezipiert, der das unmittelbare Verstehen zwischen Menschen bezeichnet (Bohnsack 2007: 60). Auf der Grundlage seiner ethnografischen Technik- und Wissenschaftsforschung zeigt Latour (2002), dass die Dinge den Menschen Handlungsmöglichkeiten eröffnen, die sie ohne sie nicht hätten (auch Latour 2010). Zwar betont er, dass Artefakte allein nicht Träger von Handlungen sind, sondern sie nur dann eine Bedeutung für das Soziale 
haben, wenn sie mit Menschen bzw. die Menschen mit den Dingen eine Verbindung eingehen (sich mit ihnen assoziieren) (Latour 2002: 235). Gleichwohl entstehen nach Latour durch das Miteinander-Interagieren von Mensch und Ding neue soziale Akteure (ebd.: 211ff.).

Um der Rolle der Dinge im Unterricht in der dokumentarischen Interpretation forschungspraktisch gerecht zu werden, integrieren wir die Frage, ob und in welcher Weise sich Menschen und Nicht-Menschen in Unterrichtssituationen miteinander assoziieren und als neuer Akteur an der Interaktion teilhaben, in die sequenzielle Analyse von Unterrichtsvideografien. Dabei gehen wir von der sequenziellen Gesprächsanalyse aus, wie sie für die dokumentarische Interpretation verbaler Daten üblich ist (Przyborski 2004; Bohnsack 2007: 121ff.). Im Interpretationsschritt der formulierenden Interpretation, in dem der immanente Sinngehalt von Äußerungen und Handlungen formuliert wird, wird zusätzlich zur Frage, welche Themen besprochen werden und welche Aktionen ausgeführt werden, auch danach gefragt, welche Dinge rekrutiert werden. Mit dem Vollzug eines Wechsels der Analyseeinstellung vom immanenten auf den dokumentarischen Sinngehalt geht es in der reflektierenden Interpretation neben der Frage, wie die Themen in der sprachlichen, diskursiven Auseinandersetzung bearbeitet werden und wie körperlich agiert wird, auch darum, wie die sich Menschen und Dinge assoziieren. Die beiden Forschungsschritte ermöglichen einen empirischen Zugriff auf das kommunikative und konjunktive Wissen der Akteure und dessen Genese in konjunktiven Erfahrungsräumen.

\section{Empirische Analysen: Historisches Lernen mit Quellen}

Quellenarbeit, d.h. die Arbeit mit historischen Zeugnissen, ist konstitutiv für den Geschichtsunterricht (z.B. Sauer 2012; Pandel 2003). Hintergrund ist die fachdidaktische Überzeugung, dass sich nur durch selbsttätige, analytisch-kritische Deutung der Vergangenheit unter Rückgriff auf Quellen ein ,reflektiertes und (selbst-) reflexives Geschichtsbewusstsein“ (Schreiber 2002) der Schülerinnen und Schüler entwickeln kann. Nur so ist die Bedingung der Möglichkeit historischer Bildung durch Geschichtsunterricht gegeben. Im Unterricht dominieren Text- und Bildquellen (vornehmlich in Form von Fotokopien oder Schulbuchmaterialien), Sachquellen, d.h. historische Originalgegenstände, spielen im Unterricht hingegen kaum eine Rolle (Heese 2007; Schneider 2010; Reeken 2003).

\subsection{Lernen mit gegenständlichen Quellen}

In einer im Rahmen eines Forschungsprojektes zur Quellenarbeit im Geschichtsunterricht (Spieß 2014) videografierten Unterrichtsstunde aus einer Einheit zur Nachkriegszeit arbeiteten Schülerinnen und Schüler einer 10. Klasse eines Gymnasiums in Gruppen mit einem kleinen Korpus an Sachquellen, das aus verschiedenen Lebensmittelmarken, Bezugsausweisen und einer Aufbewahrungsmappe bestand. Die Quellen wiesen charakteristische Gebrauchsspuren auf, die Rückschlüsse auf die historische Handlungspraxis - z.B. das Abtrennen von Markenabschnitten bei der Lebensmittelausgabe (Husumer Marke, Abb. 1) - zulassen. Der offene Arbeitsauftrag sah vor, diese historische Hand- 
lungspraxis in einer selbstständigen Auseinandersetzung mit den Quellen und in Bezug auf Vorwissen zu rekonstruieren und Rückschlüsse auf den historischen Kontext zu ziehen. Wo dies nicht gelang, sollten Fragen zu den Artefakten formuliert werden.

Abbildung 1: Husumer Lebensmittelmarken für „Vollselbstversorger“

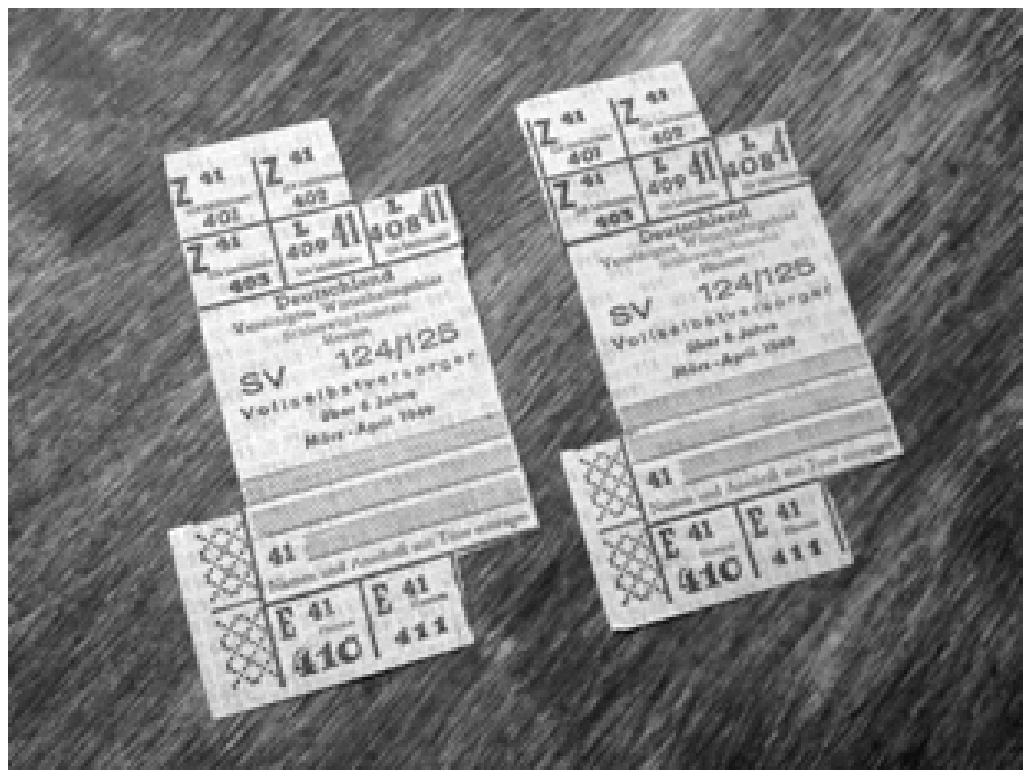

Der propositionale Gehalt der Sachquellen, als Überresten aus einer vergangenen, fremden Zeit, denen aber dennoch Bedeutung für unsere Gegenwart zukommt, wird von den Schülerinnen enaktiert: Die Jugendlichen, die um den Tisch versammelt sind, richten ihre Blicke umgehend auf die Gegenstände aus, als der Lehrer sie auf dem Tisch platziert. Anne, hinten links im Bild (Abb. 2), beginnt unmittelbar nach der Platzierung ein inhaltliches Gespräch, in dem die Gruppe sich selbstläufig, ca. zwanzig Minuten lang, auf die Artefakte bezieht:

Af was is das denn.

Bf ( ) irgendwie Tickets. oder so (2) vielleicht Zugtickets?

Lm die weiteren Dinge von denen auf den Zetteln die Rede ist liegen hier

Af witzich

(00:28-00:40)

Die erste Bezugnahme auf die Dinge erfolgt durch einen sprachlichen Verweis, der Nicht-Wissen ausdrückt. Annes Körperhaltung ist dabei distanziert-betrachtend. Ihre Arme verbleiben unter ihrem nach vorn gebeugten Oberkörper. Auch auf der nonverbalen Ebene dokumentiert sich Fremdheit gegenüber den Dingen. 
Abbildung 2: Erste Betrachtung (00:30)

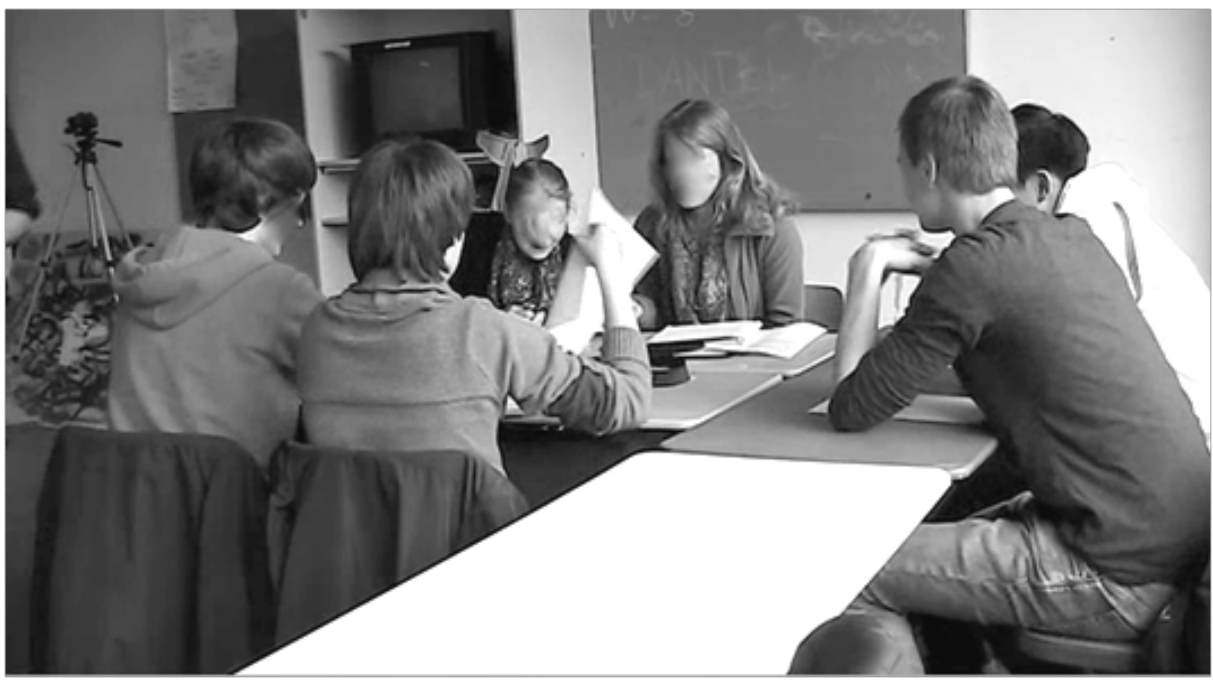

Im Folgenden elaborieren die Schülerinnen und Schüler den Arbeitsauftrag, der ebenfalls propositionalen Gehalt hat. Das Arbeitsblatt, auf dem die Aufgabe schriftlich festgehalten ist und das ebenfalls durch den Lehrer auf den Tisch gelegt wurde, wird von den Schülerinnen und Schülern in die Interaktion einbezogen und ist relevant für ihre Auseinandersetzung mit der Sache. Mit der Mutmaßung, dass es sich um Zugtickets handeln könnte, identifiziert Birthe, rechts neben Anna, die Gegenstände als Gebrauchspapiere, die einen spezifischen Gegenwert repräsentieren. Birthe geht dabei von Vorbzw. Alltagswissen aus, markiert die Identifizierung der Gegenstände aber noch als unsicher. Dem verbalen Herantasten an die Gegenstände in Form erster Hypothesen entspricht die körperliche Distanz zu den Dingen. Sie werden betrachtet, aber noch nicht berührt. Dies ändert sich im Folgenden, nachdem Carl die Vermutung aufstellt, dass es sich bei den Objekten um „Lebensmittelkarten“ handelt:

$\begin{array}{ll}\text { Cm } & \text { (eine) }{ }^{\circ} \text { Lebensmittelkarte } \\ \text { Af } & \text { echt (das is) (.) sind das nicht (.) was aus der } \\ & \text { De De (.) aus der DDR } \\ \text { Bf } & \text { Laus der DDR. ja natürlich. @(.)@ } \\ \text { Af } & \text { doch (.) wo die Lebensmitteln (verz-) jeder bekommt } \\ & \text { ja in der Woche so und so viele Sachen? } \\ \text { Cm } & \text { nee ich glaub nicht dass es DDR ist hier steht ja } \\ & \text { drauf (.) Bundesrepublik Deutschland is @(.)@ } \\ \text { Bf } & \text { @(.)@ super (.) hier steht der USA Hilfe an den } \\ & \text { wichtigsten (.) und Lebensmittelk(arte) das is } \\ & \text { schon (das) glaub ich (2) Monatsration? zehntausend } \\ & \text { Gramm? davon aus (64\% aus USA)? (4) ( } \\ \text { Af } & \text { hm hm. } \\ \text { Cm } & \text { mhm, }\end{array}$


$\mathrm{Me}$

@(.)@

(...)

Bf ich glaub das is echt so ne Lebensmittelkarte.

(3) und das hier das dann so abgeschnibbelt wurde.

(00:46-01:49)

Anne nimmt daraufhin eine alle Artefakte betreffende zeitlich-räumliche Kontextualisierung vor, indem sie deren Herkunft aus der DDR vermutet. Gegen den ironisch verneinenden Einwurf von Birthe argumentiert Anne mit ihrem historischen Wissen um die Rationierung von Konsumgütern in der DDR. Die Informationen, die die Artefakte selbst anbieten, lässt sie - zumindest vorerst - unberücksichtigt. Auf dem Video ist zu beobachten, dass eine Assoziation Annes mit den Dingen, nämlich die genauere Betrachtung der Artefakte, bis zu diesem Zeitpunkt noch nicht erfolgt ist. Die Schülerin projiziert vielmehr ihr Wissen aus der Distanz auf das Artefakt. Carl stellt von Annes Hypothese abweichend fest, dass die „Lebensmittelkarte“ aus der Bundesrepublik Deutschland stammt. Diese Feststellung basiert auf Informationen, die eines der Artefakte selbst bereithält. Birthe stimmt zu und nimmt nun selbst einen der Gegenstände in die Hand und untersucht ihn eingehend. In der Art und Weise, in der sie das Papier lediglich zwischen den Fingerspitzen hält, dokumentiert sich Vorsicht gegenüber dem Gegenstand (Abb. 3).

Birthe liest die Aufschrift auf der Rückseite der Husumer Quelle - eine Marke für Abbildung 3: Untersuchung der Husumer Marke (00:58)

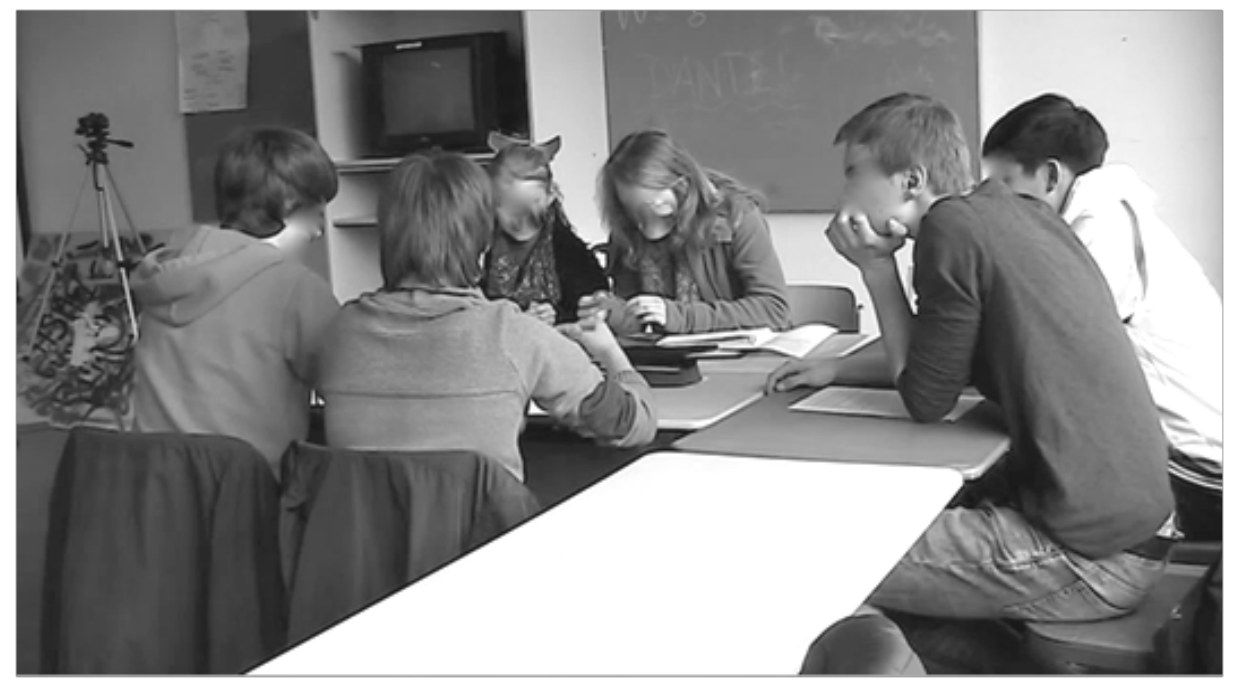

Vollselbstversorger - laut vor, wandelt dabei die durch die Gebrauchsspuren fragmentarischen Informationen leicht ab, so dass sie Sinn ergeben: 10.000 Gramm eines Lebensmittels machen eine Monatsration aus, 64\% davon stammen aus den USA. Wenngleich sowohl die Information als auch die Sinnbildung an dieser Stelle fragmentarisch bleiben, dient die so rekonstruierte Quelleninformation aber der Bestätigung der These, 
dass es sich tatsächlich um eine Lebensmittelmarke handelt. Lebensmittellieferungen durch USA-Hilfe werden damit zu einem weiteren historischen Kontext der Marke. Die Kontextualisierung erfolgt durch die Informationen, die das Artefakt nach einer ersten Inaugenscheinnahme selbst bereithält. Birthe verweist schließlich durch Zeigen auf die unregelmäßigen Ränder des Papiers und rekonstruiert aus dieser Quellenspur die historische Handlungspraxis: von den Lebensmittelmarken wurde etwas ,abgeschnibbelt".

Die Assoziation mit den Dingen führt dazu, dass aus den Artefakten selbst Informationen generiert werden. Die Ausarbeitung der Hypothesen durch die Schülerinnen und Schüler erfolgt in zirkulierenden Suchbewegungen zwischen Vor- und Dingwissen, die die gesamte Auseinandersetzung mit den Sachquellen bestimmen. Das Ding selbst erweitert in diesem Prozess das Wissen der Schülerinnen und Schüler. Im weiteren Verlauf kommt es zu unterschiedlichen, teilweise in der Sache divergierenden Interpretationen der Quellen. Ein geteilter Orientierungsrahmen der Gruppe lässt sich jedoch als Anerkennung des Nicht-Wissens über die Gegenstände und der gemeinsamen Suche nach Erkenntnissen rekonstruieren. Dabei nutzen die Schülerinnen und Schüler das in diesem Prozess kontrovers vorgetragene Wissen im Rahmen eines (Re-)Konstruktionsprozesses mit dem Ziel der Kohärenzbildung über die Identität der Artefakte als Lebensmittemarken. Dies geschieht am Ende der obigen Sequenz in Form einer ersten Zwischenkonklusion, die sich auf der nonverbalen Ebene auch dadurch zeigt, dass Birthe die untersuchte Quelle aus der Hand legt. Die Identität der Artefakte als Lebensmittelkarten wird im Folgenden erhärtet und diese Interpretation schließlich von allen Gruppenmitgliedern geteilt, so dass gegen Ende der Gruppenarbeitsphase mit Bezug auf den zeitlichen Kontext der Quellen festgehalten wird:

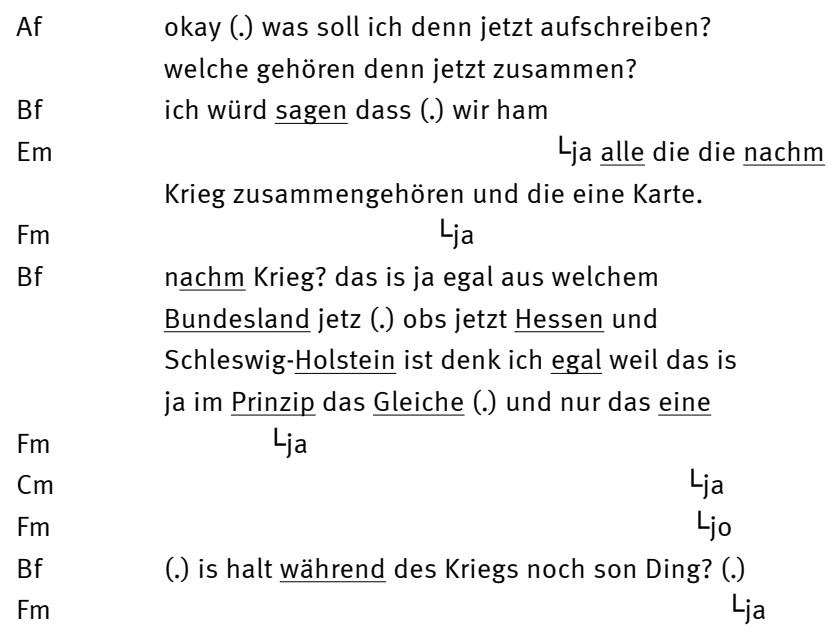

$(17: 16-17: 34)$

Die Ergebnissicherung in Form des Aufschreibens, eine Assoziation der schreibenden Schülerin mit dem Papier und dem Schreibwerkzeug, kann als Konklusion der Interaktionseinheit angesehen werden. Dabei wird deutlich, dass die Gruppe über den Vergleich 
der Artefakte unterschiedlicher geografischer Herkunft auf eine allgemeine historische Praxis der Nachkriegszeit abstrahiert: „Das is ja im Prinzip das Gleiche“. Im Sinne des Arbeitsauftrags erarbeiten die Schülerinnen und Schüler hier Informationen über die Zeit, aus der die Quellen stammen. Dabei liefert ihnen das Lehr-Lernarrangement, die Beschäftigung mit den Dingen und der offene Arbeitsauftrag, den Kontext für eine Abstraktion, die in ständigem Austausch darüber entsteht, was ausgehend von den Gegenständen behauptet werden kann und was nicht. Während die Gruppe im Rahmen der zirkulierenden Suchbewegung zwischen Vor- und Dingwissen zu einer Kohärenzbildung über die Identität und historische Funktion der Lebensmittelmarken im Allgemeinen kommt, wird dies in Bezug auf den historischen Begriff „Vollselbstversorger“, der im Text der Lebensmittelmarke erwähnt wird, nicht erreicht, auch wenn die Gruppe bemüht ist, die Bedeutung des Begriffs und davon ausgehend den historischen Kontext zu klären. Auch in dieser Sequenz wird deutlich, wie der propositionale Gehalt, der vom Ding ausgeht, die Auseinandersetzung der Schülerinnen und Schüler mit der Sache, der Lebensmittelknappheit in den Nachkriegsjahren, vorantreibt:

Bf

Af

$\mathrm{Bf}$

Af

Bf

Af

$\mathrm{Bf}$

Af

$\mathrm{Bf}$

Af

Bf

Af

$\mathrm{Bf}$

Af

Em

Af

Bf

Af

Bf

Af

$\mathrm{Bf}$
Selbstversorger vielleicht warn das Bauern die

(.) was weiß ich drei Kühe hatten (.) sieben

Schweine (.) und (.) fünf Hühner, (.) weiß ich

auch nich, @(.)@okay? (.) Und dann hatten die

$L_{k a n n}$ das nich sein dass die (.) äh die

halt (.) die brauchten ja auch irgendwas (.)

Selbstversorger

anderes noch (.) zum Beispiel Brot oder so was

Lkann das (.) kann das nicht sein

oder (.) Getreide

dass die das verabgegeben haben und verkauft

haben (.) und ähm

häh das wär ja mega blöd.

das wär überhaupt nich doof.

doch.

weil wenn (.) irgendwoher müssen die ja die Milch auch

nehmen.

ach so (.) ja. stimmt okay.

dass das die Leute sind die (.) die

Selbstversorger die (.) zum Beispiel jetzt

beispielsweise Kühe hät- haben (.) Und dann (.)

Milch von den Kühen abgeben

oder das is ne Strafe. (...)

Lwenn das ne

(.) das is ja von der USA die wollte ja helfen.

Strafe sein könnte man das ja zerkniggeln (.)

(.) das steht ja auch als Hilf- (.) dahinter

zerkniggeln. (.) zerknittern (.) zerreißen

steht ja (.) das is eine Hilfe, (.) von den (.) 
der USA,

(13:56-15:03)

Birthe äußert ein alltägliches Verständnis des Begriffs „Selbstversorger“. Als Beispiel nennt sie einen Bauern, der - da er selbst Lebensmittel erzeugt - nicht in dem Maß auf Unterstützung angewiesen ist, wie der Rest der Bevölkerung. Dennoch müsse er bestimmte andere Produkte, die er nicht selbst produziert, fremdbeziehen. Noch während Birthe ihre Deutung vorbringt, setzt auch Anne zu einer Interpretation an, mit der sie Birthes Deutung um den Aspekt der Abgabe eigener Produkte durch die Selbstversorger erweitert. Die darauf folgende Meinungsverschiedenheit versucht Anne durch den Hinweis zu beenden, dass die Lebensmittel, die ausgegeben werden, schließlich auch irgendwie produziert werden mussten. Dass es sich bei der Deutung um eine vorläufige Hypothesenbildungen im Rahmen einer Suchbewegung handelt, machen beide Schülerinnen sprachlich deutlich (,vielleicht warn das Bauern“ bzw. „kann das nicht sein dass"). Emil liefert eine weitere Hypothese, wonach der Zwang, sich selbst zu versorgen, eine zeitlich begrenzte Strafe gewesen sei. Für Birthe steht dies im Widerspruch zum Wissen über die Hilfeleistungen der USA, das bereits aus den Quellen entnommen wurde und in den zirkulierenden Suchprozess eingeflossen war. Dies wird auch bei der Betrachtung der körperlichen Bezugnahme auf die Dinge deutlich: Während Birthe Emils Deutungsversuch zurückgelehnt und mit ihren Armen vor dem Körper verschränkt zur Kenntnis nimmt und sich so auch körperlich davon distanziert (Abb. 4), beugt sie sich wenig später nach vorne und deutet mit ihrer Hand auf das Artefakt (,,dahinter steht ja (.) das ist eine Hilfe"). Anne unterstützt Birthe, indem sie ebenfalls von der Quelle ausgehend argumentiert und darauf hinweist, dass eine von Emil angenommene Strafe ins Leere laufe, wenn die betreffende Person ihren Schein einfach vernichte.

Abbildung 4: Selbstversorgung als Strafe (14:46)

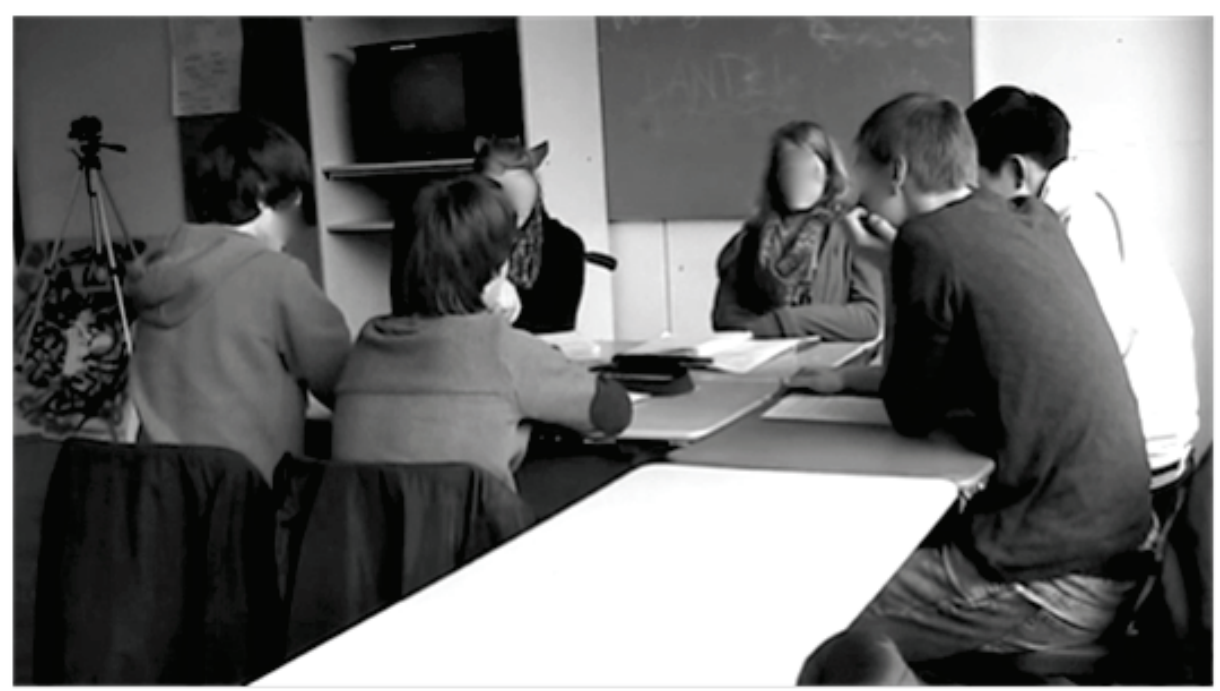


Die Gruppe kann die Unklarheit in Bezug auf den Begriff nicht lösen. Diese Kontroverse wird rituell konkludiert, indem sich die Gruppe auf einer anderen Ebene der Auseinandersetzung mit der Sache einer gemeinsamen Orientierung versichert, die darin besteht, dass die Unklarheiten akzeptiert und in Form einer Frage festgehalten werden. Während die Schülerinnen und Schüler hier divergente Positionen im Bezug auf die historische Bedeutung der Quelle vertreten, dokumentiert sich ein geteilter Orientierungsrahmen im Bezug auf den Umgang mit Quellen und der Rekonstruktion von Wissen über einen historischen Kontext. In diesem Erkenntnisprozess ist es möglich, dass Quellen unterschiedlich interpretiert werden können und Fragen offen bleiben:

$\begin{array}{ll}\text { Af } & \text { dann müssen wir jetzt eben die Fragen aufschreiben. (5) } \\ \text { Em } & \text { dieses mit dem Selbstversorgerschein? } \\ & \text { was ist unter Selbstversorgung zu verstehen? } \\ \text { Af } & \text { vor allem was hat man denn davon wenn man } \\ & \text { Selbstversorger is wenn man das auch so bekommen } \\ \text { Bf } & \text { könnte? }\end{array}$

$(17: 16-17: 34)$

Es zeigt sich, dass die Gruppe auch am Ende der Bearbeitungszeit die Offenheit des Arrangements und der Quellen noch akzeptiert und in der Lage ist, mit der Unsicherheit konstruktiv und ohne Frustration umzugehen: Fragen dürfen offen bleiben, obwohl man sich bereits über längere Zeit mit den Quellen befasst hat. In dieser Sequenz hat sich gezeigt, dass die Dinge eine intensive und lang anhaltende inhaltliche Auseinandersetzung auslösen.

\subsection{Abstraktion der Ergebnisse im kontrastiven Fallvergleich}

Der durch das schulische Lehr-Lernarrangement ermöglichte Umgang mit den Artefakten trägt in zweierlei Weise zum fachlichen Kompetenzerwerb der Schülerinnen und Schüler bei: Zum einen erschließen sie sich ein Verständnis für die Lebenswelt der Nachkriegszeit - Mangel, Rationierung von Lebensmitteln als allgemeine Phänomene sowie die Rolle der USA werden herausgearbeitet. Dabei regt das Arrangement dazu an, inhaltliche Offenheit nicht zu übergehen, sondern sie in Form von Fragen festzuhalten und zum Ausgangspunkt für weitere Recherchen und eine weitere Beschäftigung mit der Thematik in den darauffolgenden Unterrichtsstunden zu nutzen. Zum anderen ermöglicht das Lehr-Lernarrangement den Schülerinnen und Schülern, ihre historischen Methodenkompetenzen (Körber, Schreiber \& Schöner 2007) zu entwickeln und zu zeigen: Am Ausgangspunkt stehen erste, auf Vorwissen basierende Vermutungen und Hypothesen. Diese werden in einer zirkulierenden Bezugnahme auf die Sachquellen und die von ihnen bereitgehaltenen Informationen ausdifferenziert und nähern sich dabei historischen Aussagen an. Die Schülerinnen und Schüler untersuchen die Quellen eingehend. Sie gehen dabei mit großer Vorsicht und Sorgfalt vor und reagieren damit auf die spezifische Materialität und das Alter der Quellen. Sie rekonstruieren die fragmenta- 
rischen Quelleninformationen sowohl auf der Ebene der Inskriptionen als auch auf der Ebene der historischen Gebrauchsspuren. Dieser Prozess führt in teilweise kontroversen Deutungsaushandlungen zu einer historischen Sinnbildung über die historische Handlungspraxis und die sie rahmenden historischen Kontexte. Die Offenheit der Quellen regt die Schülerinnen und Schüler immer wieder zu neuen Interpretationsversuchen an. Diese erfolgen nicht willkürlich, sondern im Prozess zunehmend auf der Basis der Quellen. Als triftige Aussagen gelten in der Auseinandersetzung nur jene, die durch die Quellen bestätigt werden bzw. die durch sie nicht eindeutig widerlegt werden können (Koselleck 1989, Vetorecht der Quellen). Ziel ihres (Re-)Konstruktionsprozesses ist die Kohärenzbildung über die Identität der Artefakte. Als ein geteilter Orientierungsrahmen der Gruppe kann die Suche nach Erkenntnissen über die Vergangenheit rekonstruiert werden. Im Sinne forschenden oder entdeckenden Lernens legen es die Dinge nahe, dass Schülerinnen und Schüler neues Wissen konstruieren und fachliche Kompetenzen anwenden und einüben.

Die besondere Qualität dieses Erkenntnisprozesses zeigt sich im kontrastierenden Fallvergleich, der hier aus Platzgründen nicht mit empirischen Material veranschaulicht werden kann: In einer anderen 10. Klasse eines Gymnasium wird ebenfalls Mangel und Rationierung als alltagsgeschichtliches Thema behandelt. Die Schülerinnen und Schüler erhalten vom Lehrer eine Text- und eine Bildquelle (beide auf ein Arbeitsblatt kopiert) mit dem Auftrag, ,einen kurzen Vortrag über den Mangel an Lebensmitteln und Rohstoffen im Ersten Weltkrieg“" vorzubereiten. Dieser schriftliche Auftrag wird durch den Lehrer mündlich dahingehend präzisiert, dass die Schülerinnen und Schüler ,aus den Quellen“ (Tagebucheinträge zum Kriegsalltag in einer deutschen Stadt und ein Plakat, das zur Metallspende aufruft) etwas über ihr Thema „herausfinden“" sollen. Sie sollen auf einer Folie Notizen machen und anschließend berichten, ,wie die Ernährungssituation ist, wie die Situation der Frauen ist, wie die Situation der Jugend ist". In der Arbeitsphase liest eine Gruppe von Schülern zunächst die Tagebucheinträge. Beim Herausschreiben folgen sie der Chronologie der Tagebucheinträge, fassen die Tagebuchsätze schlagwortartig zusammen und reproduzieren damit sowohl die Gliederung der Quelle als auch deren Inhalte. Eine Auseinandersetzung um die Quellen als Dinge, die darin überlieferten Inhalte oder um die erschließbaren Kontexte findet nicht statt. Quelleninhalt und historischer Kontext werden vielmehr performativ gleichgesetzt, indem die Schüler die Quelle als abbildhafte Beschreibung der historischen Wirklichkeit behandeln, die tatsächlich Auskunft darüber geben kann, wie es wirklich gewesen ist (Ranke).

Die Interaktion in der Gruppe ist auf das vom Lehrer erwartete Produkt orientiert und kreist dementsprechend um Arbeitsverteilung und Fragen der konkreten Formulierung der Stichwortsätze für die Folie. In dieser Gruppenarbeitsphase ist zu beobachten, dass die Quellen selbst (anders als in obigen Beispiel die Sachquellen) im Vergleich zur Aufgabe nur einen nachgeordneten propositionalen Gehalt entfalten. Der Umgang mit kopierten Texten erscheint derart routiniert, dass die Schüler sich bei der Bearbeitung ausschließlich an der Effizienz ihres Handelns ausrichten, ohne die Gegenstände und die Sache, die sie repräsentieren, in diese Überlegungen einzubeziehen. Die allgemein unterrichtsspezifischen Routinen der Textbearbeitung überlagern hier die fachlichen Aneignungsprozesse. Letztere erscheinen lediglich als eine Informationsentnahme aus den Quellen (auch Spieß 2014). 
Die rekonstruierten Unterschiede zwischen der Bearbeitung von originalen Sachquellen und kopierten Textquellen lassen sich zweifach erklären: Zum einen wirkt hier die Strukturierung des Lehr-Lernarrangements. Der Geschichtslehrer im ersten Beispiel hat ein offenes Setting gestaltet, in dem weder Aufgabenstellung noch die Quellen selbst eine spezifische inhaltliche Frage aufoktroyieren. Der Lehrer im zweiten Beispiel regt hingehen durch die recht geschlossene Aufgabe mit ihrer klar vorgegebenen inhaltlichen Perspektive (Ernährungslage, Frauen, Jugend) geradezu dazu an, die Quellen als Informationsquellen zur Beschreibung eines allgemeinen historischen Sachverhalts zu verwenden. Mit der Folge, dass es durch die Schüler zu einer unzulässigen Gleichsetzung von Quelle und Sachverhalt kommt. Zum anderen können die im Fallvergleich rekonstruierten unterschiedlichen Aneignungsformen der Quellen auf die je spezifische Materialität zurückgeführt werden, in der Vergangenheit im Unterricht repräsentiert wird. Die Originale enthalten einen (fach-)spezifischen propositionalen Gehalt, an den die typischen fachunspezifischen Routinen der Textbearbeitung und Aufgabenerledigung nicht im selben Maße anschlussfähig sind wie an kopierte Textquellen. Im Vergleich sind die Sachquellen durch ihre (haptisch und visuell) erfahrbare fragmentarische Überlieferung deutungsoffener. Diese Dingqualität wird von den Schülerinnen und Schülern als besondere Deutungsbedürftigkeit enaktiert. Im Ergebnis ermöglichen die Sachquellen eine intensivere und auch genuin historische Auseinandersetzung mit dem Unterrichtsgegenstand.

\section{Theoretischer Ertrag: Lernen als zirkulierende Referenz}

Die Beschreibung von Erkenntnisprozessen als ,zirkulierende Referenz“ basiert auf Latours Untersuchungen wissenschaftlicher Praxis. Es geht also ursprünglich um wissenschaftliche Erkenntnis und ihre spezifische Bedingtheit. Vordergründig ist den von Latour beschriebenen Erkenntnisprozessen und den von uns beschriebenen Lernprozessen zwar die offensichtliche Bezugnahme auf die Dinge und deren interaktive Bedeutung gemeinsam. Gleichwohl muss man feststellen, dass zwischen den Prozessen und ihren Bedingungen auch erhebliche Unterschiede bestehen. So erschien es in unseren Unterrichtsanalysen z.B. häufig auf den ersten Blick so, als würden die Schülerinnen und Schüler auf der Basis des Umgangs mit oder der Beobachtung und Beschreibung von Gegenständen im Unterricht Wissen erwerben. In der Rekonstruktion der impliziten Sinngehalte der unterrichtlichen Interaktion mit Hilfe der Dokumentarischen Methode stellte sich allerdings heraus, dass im Unterricht die Dinge vielfach nicht der Generierung neuen Wissens im Sinne der von Latour beschriebenen Tätigkeit des Abstrahierens dienen. Vielmehr konnten wir häufiger Bezugnahmen auf Dinge im Unterricht rekonstruieren, bei denen schulisch relevantes und vielfach von den Schülerinnen und Schülern bereits gewusstes Wissen lediglich veranschaulicht wurde. Solche Bezugnahmen haben wir als Tätigkeiten des Konkretisierens bezeichnet (Asbrand, Martens \& Petersen 2013). Zwar handelt es sich auch bei der Tätigkeit des Konkretisierens um einen Prozess der zirkulierenden Referenz im Sinne Latours, da auch die Prozesse des Veranschaulichens eine gleichzeitige Bezugnahme auf die Dinge und das bereits für die Schülerinnen und Schüler verfügbare begriffliche Wissen beinhalten. Gleichzeitig aber fehlt die für 
Latours Logik der Erkenntnisgewinnung zentrale Tätigkeit des Abstrahierens, also die Erweiterung, Reorganisation und Neuschöpfung von Wissen.

Obwohl dieser Modus des Dinggebrauchs in unseren Beobachtungen häufig auftrat und somit eine nachgeordnete Positionierung von Dingen als eher typisch für schulische Lernprozesse gelten kann, konnten wir mit dem obigen Unterrichtsbeispiel zum Umgang mit Sachquellen zeigen, dass auch unterrichtliche Lernprozesse Strukturähnlichkeiten zu den von Latour beschriebenen wissenschaftlichen Erkenntnisprozessen aufweisen können: Gemeinsam ist den unterrichtlichen und wissenschaftlichen Erkenntnisprozessen, dass aus Sicht der Akteure (hier die Schülerinnen und Schüler bzw. Wissenschaftlerinnen und Wissenschaftler) neues, ihnen bisher nicht bekanntes Wissen generiert wird. In der oben beispielhaft dargestellten Sequenz mit den Sachquellen lassen sich vielfältige Mensch-Ding-Assoziationen zwischen den Schülerinnen und Schülern sowie den Sachquellen beobachten und deren Funktion für den historischen Erkenntnisprozess konnte rekonstruiert werden. Im Rahmen von zirkulierenden Suchbewegung zwischen Vorwissen der Schülerinnen und Schüler und den Informationen, die die Artefakte in der genaueren Untersuchung preisgaben, gelangten die Jugendlichen zu einer vom einzelnen Artefakt abstrahierenden historischen Deutung des Gesamtzusammenhangs. Die gleichzeitige Verfügbarkeit von historisch und geografisch ungleichen Dingen ermöglichte den Schülerinnen und Schülern, was Latour (2002) einen ,gleichen einenden Blick“ (ebd.: 50) nennt. Zwar stellten die Schülerinnen und Schüler - anders als dies eine Wissenschaftlerin tun würde - das ,synoptische Tableau“ (ebd.) aus den unterschiedlichen Quellen nicht selbst durch Recherche und Archivstudien her, die rekonstruierten Prozesse im Umgang mit den so zur Verfügung gestellten Dingen waren jedoch denen vergleichbar, die Latour beschreibt. Wie die Rekonstruktion zeigt, waren die Schülerinnen und Schüler bei der Untersuchung der Quellen implizit an der Frage orientiert, ,wie kommen wir vom Nichtwissen zum Wissen über die Gegenstände?` (auch Latour 2002: 42). Die Erkenntnis erfolgte durch Abstraktionsleistungen auf der Grundlage der Dinge. Die Wissensgenese der Schülerinnen und Schüler lässt sich hier als Abduktion beschreiben: Die Assoziationen von Menschen und Dingen ermöglichten die Herstellung eines Wissens, das mehr ist als die Summe des in der Gruppe der Menschen vorhandenen Vorwissens und des in die Quellen eingeschriebenen Wissens über die Vergangenheit. Insofern zeigt sich, dass die Schülerinnen und Schüler in Assoziation mit den Dingen zu neuen, historisch kompetenten Akteuren des Unterrichts wurden. 


\section{Autorenangaben}

\author{
Dr. Matthias Martens (Ansprechpartner) \\ Goethe-Universität Frankfurt am Main \\ Fachbereich Erziehungswissenschaften \\ m.martens@em.uni-frankfurt.de
}

\author{
Prof. Dr. Barbara Asbrand \\ Goethe-Universität Frankfurt am Main \\ Fachbereich Erziehungswissenschaften \\ b.asbrand@em.uni-frankfurt.de
}

\author{
Dr. Christian Spieß \\ Universität Osnabrück \\ Didaktik der Geschichte \\ christian.spiess@uni-osnabrueck.de
}

\section{Literatur}

Asbrand, B./Martens, M./Petersen, D. (2013): Die Rolle der Dinge in schulischen Lehr-Lernprozessen. In: Nohl, A.-M./Wulf, C. (Hrsg.): Mensch und Ding. Die Materialität pädagogischer Prozesse. Beiheft der Zeitschrift für Erziehungswissenschaft 16,2, S. 171-188.

Asbrand, B./Nohl, A.-M. (2013): Lernen in der Kontagion: Interpretieren, konjunktives und aktionistisches Verstehen im Aufbau gegenstandsbezogener Erfahrungsräume. In: Loos, P./ Nohl, A.-M./Przyborski, A./Schäffer, B. (Hrsg.): Dokumentarische Methode. Grundlagen Entwicklungen - Anwendungen. Opladen: Barbara Budrich, S. 153-169.

Blumer, H. (1969): Symbolic interactionism. Englewood Cliffs, NJ: Prentice Hall.

Bohnsack, R. (2007): Rekonstruktive Sozialforschung. Einführung in qualitative Methoden. Opladen: Barbara Budrich.

Bohnsack, R. (2009): Qualitative Bild- und Videointerpretation. Opladen: Barbara Budrich.

Breidenstein, G. (2006): Teilnahme am Unterricht. Ethnographische Studien zum Schülerjob. Wiesbaden: VS-Verlag.

Fetzer, M. (2010): Reassembling the Social Classroom. Mathematikunterricht in einer Welt der Dinge. In: Brand, B./Fetzer, M./Schütte, M. (Hrsg.): Auf den Spuren Interpretativer Unterrichtsforschung in der Mathematikdidaktik. Münster u.a.: Waxmann, S. 267-290.

Heese, T. (2007): Vergangenheit „begreifen“. Die gegenständlichen Quellen im Geschichtsunterricht. Schwalbach/Ts.: Wochenschau.

Kalthoff, H./Roehl, T. (2011): Interobjectivity and interactivity: Material objects and discourse in class. In: Human Studies 34,4, S. 451-469.

Körber, A./Schreiber, W./Schöner, A. (Hrsg.) (2007): Kompetenzen Historischen Denkens. Ein Strukturmodell als Beitrag zur Kompetenzorientierung in der Geschichtsdidaktik. Neuwied: Ars Una.

Koselleck, R. (1989): Vergangene Zukunft. Zur Semantik geschichtlicher Zeiten. Frankfurt am Main: Suhrkamp.

Krummheuer, G./Naujok, N. (1999): Grundlagen und Beispiele interpretativer Unterrichtsforschung. Opladen: Leske + Budrich.

Langeveld, M. J. (1955): Das Ding in der Welt des Kindes. In: Zeitschrift für Pädagogik 1, S. $69-83$.

Latour, B. (2002): Die Hoffnung der Pandora. Frankfurt am Main: Suhrkamp. 
Latour, B. (2005): Reassembling the social. An introduction to Actor-Network-Theory. Oxford: UP. Latour, B. (2010): Eine neue Soziologie für eine neue Gesellschaft. Frankfurt am Main: Suhrkamp. Mannheim, K. (1980): Strukturen des Denkens. Frankfurt a.M.: Suhrkamp.

Martens, M./Asbrand, B. (2009): Rekonstruktion von Handlungswissen und Handlungskompetenz - auf dem Weg zu einer qualitativen Kompetenzforschung. In: Zeitschrift für Qualitative Forschung 10,2, S. 223-239.

Martens, M./Petersen, D./Asbrand, B. (2014): Die Materialität von Lernkultur: Methodische Überlegungen zur dokumentarischen Analyse von Unterrichtsvideografien. In: Bohnsack, R/Fritzsche, B./Wagner-Willi, M. (Hrsg.): Dokumentarische Video- und Filminterpretation. Methodologie und Forschungspraxis. Opladen: Barbara Budrich, S. 179-203.

Meyer-Drawe, K. (1999): Herausforderung durch die Dinge. Das Andere im Bildungsprozess. In: Zeitschrift für Pädagogik 45,3, S. 329-342.

Nentwig-Gesemann, I. (2006): Regelgeleitete, habituelle und interaktionistische Spielpraxis. Die Analyse von Kinderspielkultur mit Hilfe videogestützter Gruppendiskussionen. In: Bohnsack, R./Przyborski, A./Schäffer, B. (Hrsg.): Das Gruppendiskussionsverfahren in der Forschungspraxis. Opladen: Barbara Budrich, S. 25-44.

Nentwig-Gesemann, I./Wagner-Willi, M. (2007): Rekonstruktive Kindheitsforschung. Zur Analyse von Diskurs- und Handlungspraxis bei Gleichaltrigen. In: Wulf, C./Zirfas, J. (Hrsg.): Pädagogik des Performativen. Theorien, Methoden, Perspektiven. Weinheim: Beltz, S. 213-223.

Nohl, A.-M. (2011): Pädagogik der Dinge. Bad Heilbrunn: Klinkhardt.

Pandel, H.-J. (2003): Quelleninterpretation. Die schriftliche Quelle im Geschichtsunterricht. Schwalbach/Ts.: Wochenschau Verlag.

Petersen, D. (2015): Anpassungsleistungen und Konstruktionsprozesse beim Grundschulübergang. Wiesbaden: VS Verlag.

Petersen, D./Asbrand, B. (2014): Anpassungsleistungen von Schülerinnen und Schülern beim Übergang von der Grundschule in die weiterführenden Schulen. In: Zeitschrift für qualitative Forschung, 14,1, S. 49-65.

Przyborski, A. (2004): Gesprächsanalyse und dokumentarische Methode. Qualitative Auswertung von Gesprächen, Gruppendiskussionen und anderen Diskursen. Wiesbaden: VS Verlag.

Rabenstein, K./Wienike, J. (2013): Der Blick auf die Dinge des Lernens. Überlegungen zur Beobachtung der materiellen Dimension pädagogischer Praktiken. In: de Boer, H./Reh, S. (Hrsg.): Beobachtung in der Schule - Beobachten lernen. Wiesbaden: VS Verlag, S. 189-202.

Reckwitz, A. (2003): Grundelemente einer Theorie sozialer Praktiken. Eine soziologische Perspektive. In: Zeitschrift für Soziologie 32,4, S. 282-301.

Reeken, D. v. (2003): Gegenständliche Quellen und museale Darstellungen. In: Günther-Arndt, H. (Hrsg.): Geschichtsdidaktik. Praxishandbuch für die Sekundarstufe I und II. Berlin: Cornelsen Scriptor, S. 137-150.

Roehl, T. (2012): From witnessing to recording - material objects and the epistemic configuration of science classes. In: Pedagogy, Culture \& Society, 20,1, S. 49-70.

Sauer, M. (2012): Geschichte unterrichten: eine Einführung in die Didaktik und Methodik. SeelzeVelber: Klett/Kallmeyer.

Schäffer, B. (2001): „Kontagion“ mit dem Technischen. Zur generationsspezifischen Einbindung in die Welt medientechnischer Dinge. In: Bohnsack, R./Nentwig-Gesemann, I./Nohl, A.-M. (Hrsg.): Die dokumentarische Methode und ihre Forschungspraxis. Grundlagen qualitativer Forschung. Opladen: Leske+Budrich, S. 43-64.

Schneider, G. (2010): Gegenständliche Quellen. In: Pandel, H.-J./Schneider, G. (Hrsg.): Handbuch Medien im Geschichtsunterricht. Schwalbach/Ts.: Wochenschau Verlag, S. 541-556.

Schreiber, W. (2002): Reflektiertes und (selbst-)reflexives Geschichtsbewusstsein durch Geschichtsunterricht fördern - ein vielschichtiges Forschungsfeld der Geschichtsdidaktik. In: Zeitschrift für Geschichtsdidaktik 1, S. 7-17. 
Spieß, C. (2014): Quellenarbeit im Geschichtsunterricht. Die empirische Rekonstruktion von Kompetenzerwerb im Umgang mit Quellen. Göttingen: V+R unipress.

Stieve, C. (2008): Von den Dingen lernen. Die Gegenstände unserer Kindheit. München: Wilhelm Fink Verlag.

Wagner-Willi, M. (2008): Die dokumentarische Videointerpretation in der erziehungswissenschaftlichen Ethnographieforschung. In: Hünersdorf, B./Maeder, C./Müller, B. (Hrsg.): Ethnographie in der Erziehungswissenschaft. Methodologische Reflexionen und empirische Annäherungen. Weinheim, München: Juventa, S. 221-231.

Wettstädt, L./Asbrand, B. (2013): Unterricht im Lernbereich Globale Entwicklung. Perspektivität als Herausforderung. In: Riegel, U./Macha, K. (Hrsg.): Videobasierte Kompetenzforschung in den Fachdidaktiken. Münster: Waxmann, S. 183-197.

Wettstädt, L./Asbrand, B. (2014): Handeln in der Weltgesellschaft. Zum Umgang mit Handlungsaufforderungen im Unterricht zu Themen des Lernbereichs Globale Entwicklung. In: Zeitschrift für internationale Bildungsforschung und Entwicklungspädagogik 36, 1, S. 4-12. 